\title{
Overview of Infectious Disease Surveillance System in Japan, 1999-2005
}

\author{
Kiyosu Taniguchi, ${ }^{1}$ Shuji Hashimoto, ${ }^{2}$ Miyuki Kawado, ${ }^{2}$ Yoshitaka Murakami, ${ }^{3}$ Michiko Izumida,${ }^{4}$ \\ Akiko Ohta, ${ }^{4}$ Yuki Tada, ${ }^{1}$ Mika Shigematsu, ${ }^{1}$ Yoshinori Yasui, ${ }^{1}$ and Masaki Nagai. ${ }^{4}$
}

\begin{abstract}
BACKGROUND: In 1999 the Communicable Disease Prevention Law of Japan was completely revised into the "New" Infectious Disease Control Law, which reiterated the importance of surveillance and information dissemination and re-organized the surveillance system. This paper is an attempt to illustrate the potential impact of the new surveillance system through a description of the existing surveillance system and data before and after the revision.

METHODS: After a historical review of surveillance system in Japan, the current surveillance system is described. Data sets of actual case numbers reported and incidence rate per 1,000,000 population are compared before and after the revision.

RESULTS: Comparison of the data between the 2 periods revealed that most of the diseases have had declining trends after the new law was enacted with several exceptions. However, although no major break in continuity is observed in seriously perceived disease, in milder diseases there are striking gaps between the numbers reported in the mandatory and sentinel reporting framework. Sentinel reporting framework maintained the continuity of data without major gaps.

CONCLUSIONS: From this perspective, the new surveillance system with two different frameworks of mandatory reporting for severe diseases and sentinel reporting for milder diseases seems to be working well. But continuous efforts should be made for evaluation and improvement of surveillance system and risk communication through the research on data analysis and effective communication method. J Epidemiol 2007; 17: S3-S13.
\end{abstract}

Key words: Sentinel Surveillance, Communicable Disease, Disease Notification.

Public health surveillance is defined by the World Health Organization (WHO) as the "Systematic ongoing collection, collation, and analysis of data and the timely dissemination of information to those who need to know so that action can be taken." It is one of the essential components for infectious disease control and no doubt a starting point for them. Timely dissemination would be a key to effective surveillance. Unfortunately in Japan, however, although disease notification triggered the local response, data reported under several notification mechanisms had not been appropriately fedback to medical society and the public for many years. This leads to a decline in the motivation to report disease. In the meantime, circumstances of infectious diseases have been changing dramatically. ${ }^{1}$ Consequently, the infectious disease surveillance in Japan was no longer functioning as a tool for infectious disease control. In 1999, the Communicable Disease Prevention Law was completely revised, and the concept of "surveillance" was legally set forth and encouraged. In this paper, after historical review of infectious disease surveillance in Japan, the current surveillance system and data based on it are described to provide overview of the infectious disease surveillance system in Japan. Through these observations, the potential impact of the new surveillance system is discussed.

Received October 5, 2007, and accepted October 23, 2007.

This study was supported by a Grant-in-Aid from the Ministry of Health, Labour and Welfare, Japan, for Research on Emerging and Re-emerging Infectious Diseases.

Infectious Disease Surveillance Center, National Institute of Infectious Diseases.

2 Department of Hygiene, Fujita Health University School of Medicine.

3 Department of Health Science, Shiga University of Medical Science.

4 Department of Public Health, Saitama Medical University Faculty of Medicine.

Address for correspondence: Kiyosu Taniguchi, Division 1, Infectious Disease Surveillance Center, National Institute of Infectious

Diseases. Toyama 1-23-1, Shinjuku-ku, Tokyo 162-8640, Japan. (e-mail: tanigk@nih.go.jp)

Copyright (C) 2007 by the Japan Epidemiological Association 


\section{HISTORICAL REVIEW OF INFECTIOUS DISEASE SURVEILLANCE IN JAPAN}

Before 1999, the Communicable Disease Prevention Law which enacted in 1897 was the only legal framework for infectious disease control and gave a legal basis for 26 reportable diseases (Table 1). Legally defined communicable diseases (11) and specially designated communicable diseases (3) are expected to be reported immediately and notifiable communicable diseases (12) within 24 hours after diagnosis by medical practitioners to prefectural or designated/core city municipal public health authorities through local public heath centers. Sexually transmitted disease, Tuberculosis, and Acquired Immunodeficiency Syndrome (AIDS) have been covered by the Venereal Disease Prevention Law since 1948, the Tuberculosis prevention law since 1951 and the AIDS Prevention Law since 1989, respectively.

In 1981 the national (sentinel) infectious disease surveillance program initiated consisting of (1) sentinel surveillance for 27 common infectious diseases (Table 7), and (2) infectious agents surveillance among local public health institutes, in order to fill the gap and vacuum of the national reportable diseases based on the law. This surveillance program, however, did not have any legal basis.

In April 1999, the Communicable Disease Prevention Law, the Venereal Disease Prevention Law, and the AIDS Prevention Law were abrogated, and the Law Concerning the Prevention of Infectious Diseases and Medical Care for Patients of Infections (hereafter referred to as the Infectious Disease Control Law) was enacted. ${ }^{2}$ In this new law, infectious disease surveillance was designated as one of the important components for disease control, and the sentinel surveillance was revised and incorporated as the national epidemiological surveillance for infectious disease (NESID) in combination with national notifiable diseases. In order to strengthen the surveillance system based on notification from physicians, collection, and analysis of the incidence and the trend of infectious diseases, effective and timely feedback of such information to the general public as well as those working in medical fields are proposed.

Infectious diseases included in this law were categorized into I through IV with specific means for control based upon the public health impact of each disease. The local outbreak of psittacosis and global outbreak of Severe Acute Respiratory Syndrome (SARS) proved that the law could not provide adequate measures against vectors and animals such as restriction of importing infecting animals, control of infected animals and extermination of vectors like mosquitoes and rats. Therefore in November 2003, the law was revised to create the new category IV including zoonotic and vector-borne diseases. ${ }^{3}$ The previous Category IV infectious diseases, except those included in the new Category IV, have been placed under the new Category V.

Finally, target diseases for the law were categorized and listed as shown in Table 2-4 and 8 . These were re-organized by another revision in December 2006, but are not referred to in this paper.

\section{DESCRIPTION OF CURRENT SURVEILLANCE SYSTEM}

Surveillance flow and function required at each level are shown in the Figure 1. All physicians must report diseases of Categories IIV immediately and Va within 7 days after identification to local public health centers which are the primary level institution for disease control and prevention strategically located all over the nation. Category $\mathrm{Vb}$ disease, which includes sentinel reporting diseases, should be reported by designated sentinel medical institutions weekly or monthly with the number of clinical cases aggregated by sex and age groups. All reports should be compatible with the reporting criteria which were documented in detail for each disease including clinical and laboratory case definitions for categories I-Va and $\mathrm{Vb}$ of hospital sentinel reporting disease, and only clinical case definitions for other $\mathrm{Vb}$ sentinel reporting diseases. $^{4}$

Local public health centers are expected to enter data into the nationwide electronic surveillance system, which enables data to be shared throughout the system. All reports are analyzed, interpreted and published by various forms of tabulations and graphs with commentary text weekly or monthly at intermediate and national level infectious disease surveillance centers, as stipulated by law. However, since category Vb diseases are reported by designated sentinel medical institutions, not by all physicians, data are displayed by weekly reported number per sentinel so as to provide understanding of the epidemic situation and disease trend. In order to be consistently representative, sentinels are expected to be selected as randomly as possible, and the numbers of sentinels per public health center coverage area are determined in proportion to the population of the area. ${ }^{5}$

\section{National Surveillance system}

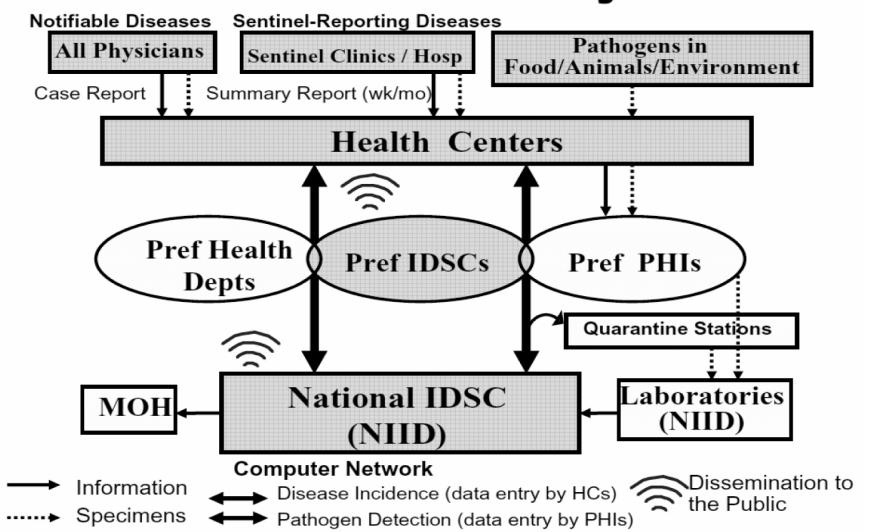

Figure 1. National surveillance structure in the national epidemiological surveillance for infectious disease(NESID). 
Table 1. Number of reported cases, mandatory reporting disease, Japan, 1993-1998.

\begin{tabular}{|c|c|c|c|c|c|c|c|}
\hline & \multicolumn{6}{|c|}{ No. of reported cases } & \multirow[b]{2}{*}{ Incidence } \\
\hline & 1993 & 1994 & 1995 & 1996 & 1997 & 1998 & \\
\hline \multicolumn{8}{|c|}{ Legally defined communicable diseases ${ }^{\dagger}$} \\
\hline Cholera & 92 & 90 & 306 & 40 & 89 & 61 & 0.90 \\
\hline Diphtheria & 5 & 1 & 1 & 1 & 1 & 3 & 0.02 \\
\hline Dysentery & 1,120 & 1,042 & 1,062 & 1,218 & 1,301 & 1,749 & 9.94 \\
\hline Meningococcal meningitis & 7 & 6 & 3 & 4 & 5 & 6 & 0.04 \\
\hline Epidemic typhus & 0 & 0 & 0 & 0 & 0 & 0 & 0.00 \\
\hline Japanese encephalitis & 8 & 6 & 4 & 6 & 6 & 4 & 0.05 \\
\hline Paratyphoid fever & 46 & 49 & 75 & 32 & 37 & 54 & 0.39 \\
\hline Plague & 0 & 0 & 0 & 0 & 0 & 0 & 0.00 \\
\hline Scarlet fever & 23 & 6 & 5 & 4 & 3 & 0 & 0.05 \\
\hline Smallpox & 0 & 0 & 0 & 0 & 0 & 0 & 0.00 \\
\hline Typhoid fever & 129 & 71 & 64 & 81 & 79 & 61 & 0.64 \\
\hline
\end{tabular}

Specially designated communicable diseases

$\begin{array}{lrrrrrrr}\text { Acute poliomyelitis } & 3 & 1 & 1 & 0 & 0 & 0 & 0.01 \\ \text { Enterohemorrhagic Escherichia coli infection } & \ldots & \ldots & \ldots & 1,287 & 1,941 & 2,077 & 14.08 \\ \text { Lassa fever } & 0 & 0 & 0 & 0 & 0 & 0 & 0.00\end{array}$

Anthrax
Filariasis
Infectious diarrhea
Influenza
Malaria
Measles
Pertussis
Rabies
Relapsing fever
Scrub typhus
Tetanus

Notifiable communicable diseases

$\begin{array}{rrrrrrr}0 & 2 & 0 & 0 & 0 & 0 & 0.00 \\ 1 & 0 & 1 & 1 & 0 & 1 & 0.01 \\ 2 & 1 & 0 & 10 & 140 & 0 & 0.20 \\ 16,655 & 2,404 & 22,393 & 8,774 & 8,816 & 14,778 & 97.98 \\ 58 & 74 & 66 & 51 & 69 & 79 & 0.53 \\ 2,002 & 1,766 & 931 & 1,640 & 899 & 761 & 10.62 \\ 131 & 145 & 226 & 183 & 42 & 43 & 1.02 \\ 0 & 0 & 0 & 0 & 0 & 0 & 0.00 \\ 0 & 0 & 0 & 0 & 0 & 0 & 0.00 \\ 712 & 652 & 529 & 423 & 487 & 538 & 4.43 \\ 33 & 44 & 45 & 44 & 47 & 47 & 0.35\end{array}$

Acquired immunodeficiency syndrome

Acquired Immunodeficiency Syndrome Prevention Law

$\begin{array}{lrllllll}\text { Asymptomatic } & 277 & 298 & 277 & 376 & 397 & 422 & 2.72 \\ \text { Acquired immunodeficiency syndrome } & 86 & 136 & 169 & 234 & 250 & 231 & 1.47\end{array}$

Chancroid

Venereal Diseases Prevention Law

Gonorrhea

Lymphogranuloma inguinale

Syphilis

...: Not applicable.

*: Incidence rate is per year per 1,000,000 population in 1993-1998

$\dagger$ : Defined by the Communicable Disease Prevention Law 
Table 2. Number of reported cases of Category I, II and III, mandatory reporting disease, Japan, 1999-2005.

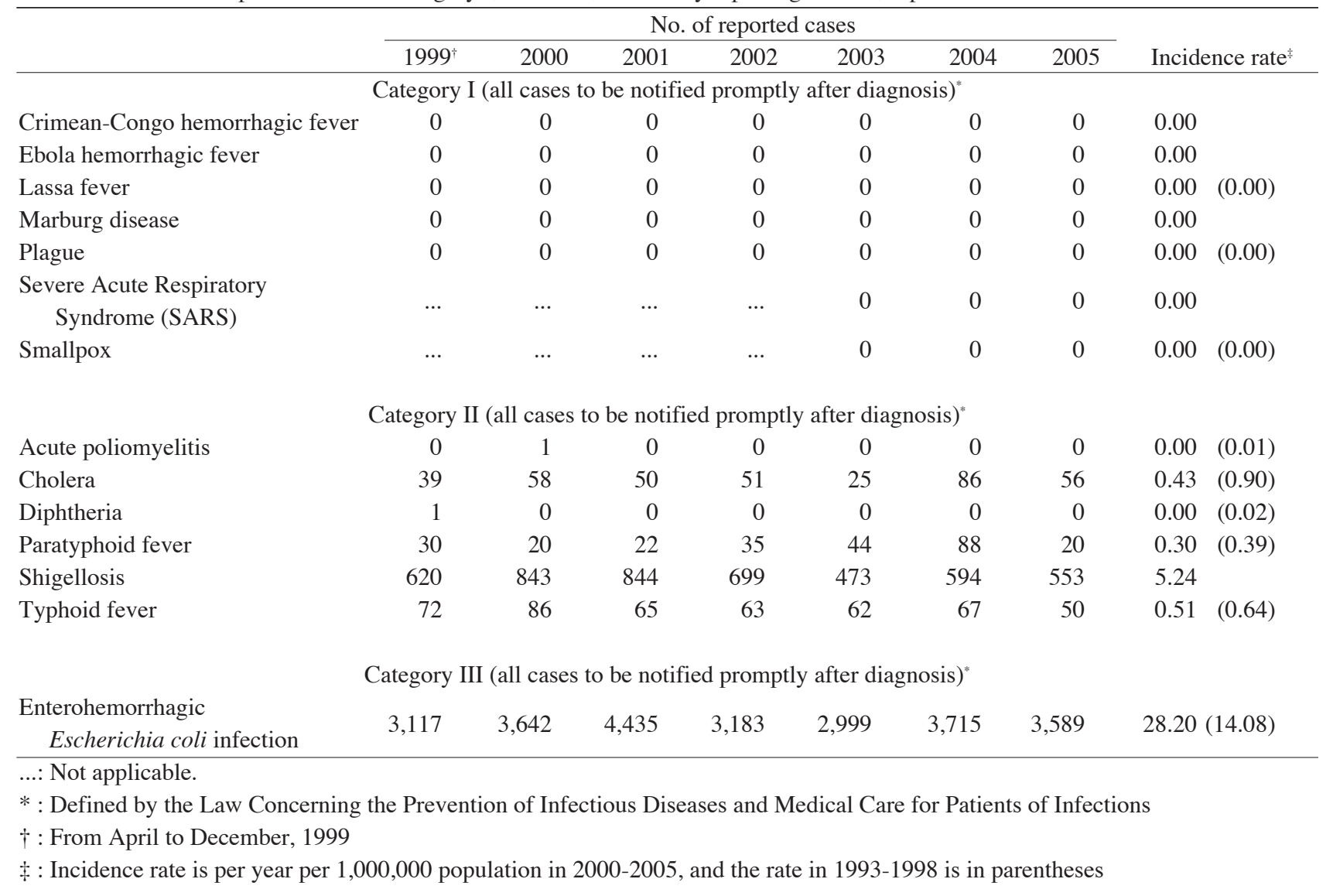


Table 3. Number of reported cases of Category IV, mandatory reporting disease, Japan, 1999-2005.*

\begin{tabular}{|c|c|c|c|c|c|c|c|c|}
\hline & \multicolumn{7}{|c|}{ No. of reported cases } & \multirow[b]{2}{*}{ Incidence rate } \\
\hline & 1999 & 2000 & 2001 & 2002 & 2003 & 2004 & 2005 & \\
\hline \multicolumn{9}{|c|}{ Category IV (all cases to be notified promptly after diagnosis)* } \\
\hline Anthrax & 0 & 0 & 0 & 0 & 0 & 0 & 0 & $0.00(0.00)$ \\
\hline Avian influenza virus infection & $\ldots$ & $\ldots$ & $\ldots$ & $\ldots$ & 0 & 0 & 0 & 0.00 \\
\hline Botulism & $\ldots$ & $\ldots$ & $\ldots$ & $\ldots$ & 0 & 0 & 3 & 0.01 \\
\hline Brucellosis & 0 & 0 & 0 & 1 & 0 & 0 & 2 & 0.00 \\
\hline Coccidioidomycosis & 0 & 1 & 2 & 3 & 1 & 5 & 5 & 0.02 \\
\hline Dengue fever & 9 & 18 & 50 & 52 & 32 & 49 & 74 & 0.36 \\
\hline \multicolumn{9}{|l|}{ Echinococcosis } \\
\hline Granulosus & 1 & 2 & 2 & 2 & 1 & 1 & 2 & 0.01 \\
\hline Multilocularis & 6 & 20 & 13 & 8 & 19 & 25 & 18 & 0.13 \\
\hline Epidemic typhus & 0 & 0 & 0 & 0 & 0 & 0 & 0 & $0.00(0.00)$ \\
\hline $\begin{array}{l}\text { Hantavirus pulmonary } \\
\text { syndrome }\end{array}$ & 0 & 0 & 0 & 0 & 0 & 0 & 0 & 0.00 \\
\hline $\begin{array}{l}\text { Hemorrhagic fever with renal } \\
\text { syndrome }\end{array}$ & 0 & 0 & 0 & 0 & 0 & 0 & 0 & 0.00 \\
\hline Hepatitis A & 761 & 381 & 491 & 502 & 303 & 139 & 170 & 2.60 \\
\hline Hepatitis E & 0 & 3 & 0 & 16 & 30 & 37 & 42 & 0.17 \\
\hline Herpes B virus infection & 0 & 0 & 0 & 0 & 0 & 0 & 0 & 0.00 \\
\hline Japanese encephalitis & 5 & 7 & 5 & 8 & 1 & 5 & 7 & $0.04(0.05)$ \\
\hline Japanese spotted fever & 39 & 38 & 40 & 36 & 52 & 66 & 62 & 0.38 \\
\hline Legionellosis & 56 & 154 & 86 & 167 & 146 & 161 & 281 & 1.30 \\
\hline Leptospirosis & $\ldots$ & $\ldots$ & $\ldots$ & $\ldots$ & 1 & 18 & 17 & 0.09 \\
\hline Lyme disease & 14 & 12 & 15 & 15 & 5 & 5 & 8 & 0.08 \\
\hline Lyssavirus infection & $\ldots$ & $\ldots$ & $\ldots$ & $\ldots$ & 0 & 0 & 0 & 0.00 \\
\hline Malaria & 112 & 154 & 109 & 83 & 78 & 75 & 67 & $0.74(0.53)$ \\
\hline Monkeypox & $\ldots$ & $\ldots$ & $\ldots$ & $\ldots$ & 0 & 0 & 0 & 0.00 \\
\hline Nipah virus infection & $\ldots$ & $\ldots$ & $\ldots$ & $\ldots$ & 0 & 0 & 0 & 0.00 \\
\hline Psittacosis & 23 & 18 & 35 & 54 & 44 & 40 & 34 & 0.29 \\
\hline Q fever & 12 & 24 & 42 & 47 & 9 & 7 & 8 & 0.18 \\
\hline Rabies & 0 & 0 & 0 & 0 & 0 & 0 & 0 & $0.00(0.00)$ \\
\hline Relapsing fever & 0 & 0 & 0 & 0 & 0 & 0 & 0 & 0.00 \\
\hline $\begin{array}{l}\text { Scrub typhus } \\
\text { (Tsutsugamushi disease) }\end{array}$ & 556 & 791 & 491 & 338 & 402 & 313 & 345 & $3.51(4.43)$ \\
\hline Tularemia & $\ldots$ & $\ldots$ & $\ldots$ & $\cdots$ & 0 & 0 & 0 & 0.00 \\
\hline West Nile fever & $\ldots$ & $\ldots$ & $\ldots$ & 0 & 0 & 0 & 1 & 0.00 \\
\hline Yellow fever & 0 & 0 & 0 & 0 & 0 & 0 & 0 & 0.00 \\
\hline
\end{tabular}

...: Not applicable.

* : Defined by the Law Concerning the Prevention of Infectious Diseases and Medical Care for Patients of Infections

$\dagger$ : From April to December, 1999

$\$$ : Incidence rate is per year per $1,000,000$ population in $2000-2005$, and the rate in $1993-1998$ is in parentheses 
Table 4. Number of reported cases of Category Va, mandatory reporting disease, Japan, 1999-2005."

\begin{tabular}{|c|c|c|c|c|c|c|c|c|}
\hline & \multicolumn{7}{|c|}{ No. of reported cases } & \multirow[b]{2}{*}{ Incidence rate } \\
\hline & 1999 & 2000 & 2001 & 2002 & 2003 & 2004 & 2005 & \\
\hline \multicolumn{9}{|c|}{ Category Va (all cases to be reported by all physicians within 7days after diagnosis) } \\
\hline \multicolumn{9}{|c|}{ Acquired immunodeficiency syndrome (AIDS) } \\
\hline Asymptomatic & 346 & 413 & 570 & 547 & 564 & 699 & 753 & $4.64(2.72)$ \\
\hline AIDS & 215 & 331 & 320 & 312 & 337 & 386 & 360 & $2.68(1.47)$ \\
\hline Other & 27 & 50 & 57 & 57 & 69 & 77 & 90 & 0.52 \\
\hline Amebiasis & 276 & 378 & 429 & 465 & 520 & 610 & 698 & 4.05 \\
\hline Acute encephalitis & $\ldots$ & $\ldots$ & $\ldots$ & $\ldots$ & 12 & 166 & 188 & 0.48 \\
\hline Congenital rubella syndrome & 0 & 1 & 1 & 1 & 1 & 10 & 2 & 0.02 \\
\hline Creutzfeldt-Jakob disease & 92 & 108 & 133 & 147 & 118 & 175 & 152 & 1.09 \\
\hline Cryptosporidiosis & 4 & 3 & 11 & 109 & 8 & 92 & 12 & 0.31 \\
\hline Giardiasis & 42 & 98 & 137 & 113 & 103 & 94 & 86 & 0.83 \\
\hline $\begin{array}{l}\text { Severe invasive streptococcal } \\
\text { infections }\end{array}$ & 22 & 47 & 47 & 92 & 53 & 52 & 60 & 0.46 \\
\hline Syphilis & 751 & 759 & 585 & 575 & 509 & 533 & 543 & $4.58(4.73)$ \\
\hline Meningococcal meningitis & 10 & 15 & 8 & 9 & 18 & 21 & 10 & $0.11(0.04)$ \\
\hline Tetanus & 66 & 91 & 80 & 106 & 73 & 101 & 115 & $0.74(0.35)$ \\
\hline $\begin{array}{l}\text { Vancomycin-resistant } \\
\text { Enterococcus infection }\end{array}$ & 23 & 36 & 40 & 44 & 59 & 58 & 69 & 0.40 \\
\hline \multicolumn{9}{|l|}{ Vancomycin-resistant } \\
\hline $\begin{array}{l}\text { Staphylococcus aureus } \\
\text { infection }\end{array}$ & ... & $\ldots$ & $\ldots$ & $\ldots$ & 0 & 0 & 0 & 0.00 \\
\hline \multicolumn{9}{|c|}{ Viral hepatitis (excluding hepatitis A and E) } \\
\hline Type B & 510 & 425 & 330 & 332 & 245 & 241 & 208 & 2.33 \\
\hline Type C & 136 & 119 & 65 & 61 & 65 & 43 & 57 & 0.54 \\
\hline Type D & 0 & 0 & 0 & 0 & 0 & 0 & 0 & 0.00 \\
\hline Other & 74 & 40 & 29 & 23 & 19 & 7 & 10 & 0.17 \\
\hline Unknown & 36 & 22 & 14 & 14 & 4 & 2 & 1 & 0.07 \\
\hline
\end{tabular}

...: Not applicable.

* : Defined by the Law Concerning the Prevention of Infectious Diseases and Medical Care for Patients of Infections

$\dagger:$ From April to December, 1999

$\$$ : Incidence rate is per year per $1,000,000$ population in $2000-2005$, and the rate in $1993-1998$ is in parentheses

Table 5. Number of sentinel medical institutions, Japan, 1993-1998.

\begin{tabular}{lrrrrrr}
\hline Sentinels $^{*}$ & 1993 & 1994 & 1995 & 1996 & 1997 & 1998 \\
\hline Pediatric disease & 2,425 & 2,425 & 2,440 & 2,412 & 2,412 & 2,412 \\
Ophthalmologic disease & 315 & 314 & 314 & 316 & 316 & 316 \\
Sexually transmitted disease & 596 & 599 & 604 & 606 & 606 & 606 \\
Hospital & 544 & 540 & 521 & 518 & 518 & 518 \\
\hline
\end{tabular}

* : Based on the national infectious disease surveillance program

Table 6. Number of sentinel medical institutions, Japan, 1999-2005.

\begin{tabular}{lrrrrrrr}
\hline Sentinels $^{*}$ & $1999^{*}$ & 2000 & 2001 & 2002 & 2003 & 2004 & 2005 \\
\hline Influenza & 4,128 & 4,585 & 4,649 & 4,698 & 4,703 & 4,653 & 4,729 \\
Pediatric disease & 2,875 & 2,978 & 3,019 & 3,036 & 3,041 & 3,019 & 3,065 \\
Ophthalmologic disease & 589 & 625 & 634 & 634 & 634 & 633 & 649 \\
Sexually transmitted disease & 855 & 897 & 911 & 917 & 920 & 916 & 931 \\
Hospital Weekly & 456 & 459 & 470 & 473 & 471 & 475 & 471 \\
& 445 & 457 & 458 & 465 & 468 & 468 & 471 \\
\hline
\end{tabular}

* : Defined by the Law Concerning the Prevention of Infectious Diseases and Medical Care for Patients of Infections

$\dagger:$ From April to December, 1999 
Table 7. Number of reported cases per sentinel, sentinel reporting disease, Japan, 1993-1998.

\begin{tabular}{|c|c|c|c|c|c|c|c|}
\hline & \multicolumn{6}{|c|}{ No. of reported cases per sentinel } & \multirow[b]{2}{*}{ Average } \\
\hline & 1993 & 1994 & 1995 & 1996 & 1997 & 1998 & \\
\hline \multicolumn{8}{|c|}{ Pediatric disease sentinel (weekly report) } \\
\hline Atypical pneumonia & 10.95 & 8.90 & 9.53 & 11.09 & 9.22 & 8.46 & 9.69 \\
\hline Chickenpox & 77.01 & 73.36 & 76.23 & 78.79 & 76.75 & 67.02 & 74.86 \\
\hline Erythema infectiosum & 7.02 & 5.29 & 5.69 & 15.62 & 22.65 & 12.72 & 11.50 \\
\hline Exanthem subitum & 35.73 & 36.76 & 35.02 & 34.69 & 35.66 & 34.92 & 35.46 \\
\hline Group A streptococcal pharyngitis & 29.02 & 32.98 & 24.55 & 31.00 & 34.77 & 33.94 & 31.04 \\
\hline Hand, foot and mouth disease & 38.15 & 22.59 & 65.04 & 10.17 & 30.98 & 52.06 & 36.50 \\
\hline Herpangina & 29.87 & 36.94 & 32.61 & 40.03 & 36.25 & 32.62 & 34.72 \\
\hline Infectious gastroenteritis & 169.23 & 165.97 & 193.20 & 162.71 & 173.88 & 161.40 & 171.07 \\
\hline Infantile vomiting and diarrhea & 36.29 & 32.48 & 47.18 & 31.43 & 34.96 & 33.19 & 35.92 \\
\hline Influenza & 262.49 & 44.77 & 310.14 & 146.27 & 162.91 & 235.39 & 193.66 \\
\hline Measles (excluding adult) & 14.25 & 8.89 & 7.32 & 9.55 & 6.49 & 4.05 & 8.42 \\
\hline Mumps & 38.04 & 52.65 & 29.05 & 46.72 & 62.22 & 56.60 & 47.55 \\
\hline Pertussis & 1.51 & 1.85 & 2.32 & 2.36 & 1.13 & 0.98 & 1.69 \\
\hline Pharyngoconjunctival fever & 3.85 & 1.76 & 4.40 & 3.43 & 2.44 & 2.30 & 5.09 \\
\hline Rubella & 60.97 & 14.79 & 6.67 & 11.10 & 19.57 & 9.14 & 20.37 \\
\hline Kawasaki disease (Clinic sentinel) & 0.46 & 0.57 & 0.55 & 0.56 & 0.59 & 0.57 & 0.55 \\
\hline \multicolumn{8}{|c|}{ Ophthalmologic disease sentinel (weekly report) ${ }^{\dagger}$} \\
\hline Acute hemorrhagic conjunctivitis & 5.67 & 20.25 & 3.68 & 1.39 & 1.28 & 1.54 & 5.63 \\
\hline Epidemic keratoconjunctivitis & 41.93 & 53.19 & 70.36 & 63.31 & 59.84 & 53.88 & 57.08 \\
\hline Pharyngoconjunctival fever & 2.03 & 2.54 & 2.49 & 2.35 & 2.15 & 2.79 & 2.39 \\
\hline \multicolumn{8}{|c|}{ Sexually transmitted disease sentinel (monthly report) ${ }^{\dagger}$} \\
\hline Condyloma acuminatum & 4.75 & 4.02 & 3.55 & 3.39 & 3.42 & 3.81 & 3.83 \\
\hline Genital chlamydial infection & 23.13 & 23.91 & 22.74 & 23.80 & 25.97 & 28.50 & 24.68 \\
\hline Genital herpes & 9.65 & 9.82 & 9.44 & 10.15 & 9.81 & 9.43 & 9.72 \\
\hline Gonorrhea & 11.28 & 10.48 & 11.06 & 13.04 & 14.19 & 16.36 & 12.73 \\
\hline Syphilis & $\ldots$ & $\ldots$ & $\ldots$ & $\ldots$ & $\ldots$ & 1.46 & 1.46 \\
\hline Trichomoniasis & 6.52 & 6.12 & 4.99 & 4.51 & 3.93 & 3.77 & 4.97 \\
\hline \multicolumn{8}{|c|}{ Sentinel hospital (monthly report) ${ }^{\dagger}$} \\
\hline Aseptic meningitis & 3.96 & 5.11 & 3.11 & 2.96 & 6.42 & 9.86 & 5.24 \\
\hline Bacterial meningitis & 0.42 & 0.44 & 0.47 & 0.50 & 0.55 & 0.46 & 0.48 \\
\hline $\begin{array}{l}\text { Encephalitis/Encephalopathy/Myelitis/ } \\
\text { Reye syndrome }\end{array}$ & 0.44 & 0.35 & 0.35 & 0.35 & 0.39 & 0.48 & 0.39 \\
\hline Hepatitis A & 1.47 & 1.32 & 0.67 & 0.67 & 0.49 & 0.26 & 0.81 \\
\hline Hepatitis B & 1.24 & 0.99 & 0.80 & 1.02 & 0.88 & 0.89 & 0.97 \\
\hline Hepatitis C & $\ldots$ & $\ldots$ & $\ldots$ & $\ldots$ & $\ldots$ & 1.74 & 1.74 \\
\hline Other hepatitis & 4.88 & 2.86 & 2.23 & 2.68 & 2.84 & 1.62 & 2.85 \\
\hline Kawasaki disease (Hospital sentinels) & 2.89 & 3.30 & 3.52 & 3.27 & 3.32 & 3.52 & 3.30 \\
\hline
\end{tabular}

...: Not applicable.

* : Average of the numbers of reported cases per sentinel in 1993-1998

$\dagger$ : Based on the national infectious disease surveillance program 
Table 8. Number of reported cases per sentinel, sentinel reporting disease, Japan, 1999-2005.

\begin{tabular}{|c|c|c|c|c|c|c|c|c|c|}
\hline & \multicolumn{7}{|c|}{ No. of reported cases per sentinel } & \multirow{2}{*}{\multicolumn{2}{|c|}{ Average $^{*}$}} \\
\hline & $1999^{\dagger}$ & 2000 & 2001 & 2002 & 2003 & 2004 & 2005 & & \\
\hline \multicolumn{10}{|c|}{ Influenza sentinel (weekly report) } \\
\hline Influenza & 15.32 & 167.93 & 65.70 & 159.01 & 247.14 & 165.50 & 330.65 & 189.32 & $(193.66)$ \\
\hline \multicolumn{10}{|c|}{ Pediatric disease sentinel (weekly report) } \\
\hline Chickenpox & 56.50 & 92.36 & 89.90 & 86.73 & 82.39 & 81.46 & 79.05 & 85.32 & $(74.86)$ \\
\hline Erythema infectiosum & 6.47 & 11.50 & 22.41 & 19.02 & 11.77 & 16.20 & 12.82 & 15.62 & $(11.50)$ \\
\hline Exanthem subitum & 33.30 & 42.57 & 41.48 & 38.43 & 38.39 & 37.53 & 34.72 & 38.85 & $(35.46)$ \\
\hline $\begin{array}{l}\text { Group A streptococcal } \\
\text { pharyngitis }\end{array}$ & 31.40 & 53.10 & 51.32 & 51.38 & 54.77 & 68.58 & 60.27 & 56.57 & $(31.04)$ \\
\hline Hand, foot and mouth disease & 17.67 & 68.96 & 42.32 & 29.98 & 56.78 & 29.39 & 28.84 & 42.71 & $(36.50)$ \\
\hline Herpangina & 53.84 & 49.45 & 46.44 & 36.71 & 48.89 & 34.94 & 47.07 & 43.92 & $(34.72)$ \\
\hline Infectious gastroenteritis & 176.55 & 297.57 & 289.58 & 293.12 & 298.19 & 315.56 & 307.32 & 300.22 & (171.07) \\
\hline Measles (excluding adult) & 2.04 & 7.57 & 11.20 & 4.11 & 2.72 & 0.51 & 0.18 & 4.38 & $(8.42)$ \\
\hline Mumps & 24.02 & 44.62 & 84.37 & 59.56 & 27.86 & 42.26 & 61.28 & 53.33 & $(47.55)$ \\
\hline Pertussis & 0.92 & 1.28 & 0.58 & 0.48 & 0.51 & 0.73 & 0.44 & 0.67 & $(1.69)$ \\
\hline Pharyngoconjunctival fever & 3.73 & 6.81 & 8.49 & 5.11 & 13.40 & 20.23 & 16.29 & 11.72 & $(5.09)$ \\
\hline $\begin{array}{l}\text { Respiratory syncytial virus } \\
\text { infection }\end{array}$ & $\cdots$ & $\cdots$ & 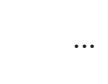 & $\cdots$ & $\S$ & $\S$ & $\S$ & $\S$ & \\
\hline Rubella & 1.03 & 1.05 & 0.85 & 0.98 & 0.92 & 1.40 & 0.29 & 0.92 & $(20.37)$ \\
\hline \multicolumn{10}{|c|}{ Ophthalmologic disease sentinel (weekly report) } \\
\hline $\begin{array}{l}\text { Acute hemorrhagic } \\
\text { conjunctivitis }\end{array}$ & 1.84 & 2.29 & 2.11 & 1.60 & 1.61 & 1.21 & 1.12 & 1.66 & $(5.63)$ \\
\hline Epidemic keratoconjunctivitis & 40.65 & 65.40 & 62.30 & 54.53 & 48.51 & 44.02 & 45.78 & 53.42 & $(57.08)$ \\
\hline \multicolumn{10}{|c|}{ Sexually transmitted disease sentinel (monthly report) } \\
\hline Condyloma acuminatum & 3.73 & 5.08 & 5.68 & 6.22 & 6.80 & 7.17 & 7.30 & 6.38 & $(3.83)$ \\
\hline Genital chlamydial infection & 29.28 & 41.28 & 44.83 & 47.73 & 45.59 & 41.65 & 37.66 & 43.12 & $(24.68)$ \\
\hline Genital herpes & 7.68 & 9.97 & 10.22 & 10.54 & 10.69 & 10.67 & 11.02 & 10.52 & $(9.72)$ \\
\hline Gonorrhea & 13.86 & 18.87 & 22.68 & 23.91 & 22.50 & 19.02 & 16.11 & 20.52 & (12.73) \\
\hline
\end{tabular}

Acute encephalitis (excluding Japanese encephalitis and West Nile encephalitis)

Aseptic meningitis

Bacterial meningitis

Chlamydial pneumonia (excluding psittacosis)

Measles in adults

Mycoplasmal pneumonia

$\begin{array}{rrrrrrrrr}0.28 & 0.32 & 0.29 & 0.23 & 0.21 & \ldots & \ldots & 0.26 & \\ & & & & & & & & \\ 2.47 & 4.08 & 2.67 & 6.31 & 3.45 & 2.16 & 1.64 & 3.39 & (5.24) \\ 0.52 & 0.56 & 0.59 & 0.63 & 0.63 & 0.80 & 0.66 & 0.65 & (0.48) \\ 0.28 & 0.39 & 0.39 & 0.52 & 0.43 & 0.51 & 0.68 & 0.49 & \\ 0.18 & 0.93 & 1.98 & 0.93 & 0.98 & 0.12 & 0.01 & 0.83 & \\ 2.49 & 4.55 & 9.07 & 9.05 & 12.08 & 12.66 & 15.03 & 10.41 & \end{array}$

Methicillin-resistant Staphylococcus aureus infection 24.92 Sentinel hospital (monthly report)

Multi-drug-resistant Pseudomonas aeruginosa infection

Penicillin-resistant Streptococcus pneumoniae infection Sentinel hospital (weekly report)

\footnotetext{
...: Not applicable.

* : Average of the numbers of reported cases per sentinel in 2000-2005, and average in 1993-1998 in parentheses

$\dagger$ : Defined by the Law Concerning the Prevention of Infectious Diseases and Medical Care for Patients of Infections

$\S$ : The cases were reported, but the number of cases per sentinel was not calculated.
} 


\section{SUMMARY OF REPORTS FROM SURVEILLANCE SYSTEM}

In order to understand the reporting situation under the current surveillance system, all the reports after the enactment of the new law were summarized in this section. To illustrate surveillance under the new law, data are compared with those under the old law.

\section{Materials and Methods}

Surveillance data in 1999-2005 based on the Infectious Disease Control Law were derived from the annual report of NESID. To compare the situation of surveillance, 6-year data sets in 19931998 based on the Communicable Disease Prevention Law are obtained from the Statistics on communicable diseases. Data for syphilis and AIDS are obtained from the statistics based on the report on the Venereal Disease Prevention Law and the annual report for the AIDS Prevention Law, respectively. Data in 1999 are only available in April to December because of the change of the law in April 1999. Finally, data sets of actual case numbers reported and incidence rate per 1,000,000 population are compared before and after the revision. Incidence rates of 1993-1998 are calculated using 1995 census population and those of 19992005 are based on the 2002 population.

In order to discuss the value of sentinel surveillance, sentinel surveillance data in 1993-1998 are extracted from the annual report of the national infectious disease surveillance program and compared with the data in 1999-2005 from the annual report of NESID. Annual case numbers per sentinel, and number of sentinels are compared for the two periods. In addition, data are compared with cases reported under the old law, where data on the same diseases are available.

\section{Secular Trend in Target Infectious Diseases}

Annual reported number and average incidence rate per 1,000,000 population through 6 years of all notifiable diseases under the old law are shown in Table 1, and data on diseases under the new law in Table 2-4. Data are compared between two periods whenever data on the relevant disease entity are available. Most of the diseases have declining trends in the new law period compared with before, although occasional increases are observed in paratyphoid fever and cholera. Exceptionally, enterohemorrhagic E-coli infection, malaria, tetanus and AIDS showed more cases under the new law. Cases with dysentery decreased under the new law, but dysentery in the old era included both disease entities caused by Shigella dysentery and entamoeba histolytica.

As to the sentinel reporting diseases, the number of sentinels under the new law increased mainly because of the change in selection criteria (Table 5, 6). Influenza sentinels in the old period are the same as pediatric ones, but are expanded in combination with internal medicine clinics mainly for adult patients under the new law. Ophthalmologic and sexually transmitted diseases sentinels increased in number, but hospital sentinels remain in the same level as no major change on selection criteria.

Under such circumstances, many sentinel reporting diseases increased in terms of the number of reports per sentinel, although they fluctuated in epidemicity (Table 7,8 ). But several vaccine preventable diseases including measles, rubella and pertussis decreased dramatically in the last 7 years. Continuity of the reported number is considered to be maintained throughout the observed period.

Influenza, measles, pertussis, scarlet fever and infectious diarrhea were mandatory notifiable diseases in the old period (Table 1), but they were monitored by the national sentinel surveillance program as well since 1981 (Table 7, 8). Comparison reveals a far greater number of reported cases in the sentinel system than in the mandatory system. This might be partly caused by the difference in case definition. Reports of influenza and infectious diarrhea in the mandatory system are based on clinical characteristics in the same manner as in the sentinel system; and reports of measles, pertussis and scarlet fever based on the clinical diagnosis were also acceptable in the mandatory system. Since there were no documented reporting criteria under the old law, reports mainly depended upon the clinician's decision irrespective of whether or not laboratory confirmation was made. Similar observations were made for syphilis, where there were 887 reports from 606 sentinels, but only 553 cases were reported from all physicians in 1998.

\section{DISCUSSION}

The Communicable Disease Prevention Law enacted in 1st April 1898 long provided the legal framework for infectious disease control in Japan. Mandatory reporting of national notifiable disease based upon this law was the only system for infectious disease surveillance. The basic policy of the law was the traditional attempt to prevent the massive expansion of infectious disease by disease notification and following isolation and quarantine. In the 1990 's, however, the circumstances surrounding infectious diseases showed drastic changes including emerging and re-emerging infectious diseases, globalization of travel and trade, animal diseases crossing into human populations, and accidental or deliberate release of biological agents. These situations made an effective outbreak response more difficult. And lacking appropriate risk communication including effective feedback of infectious disease information, clinicians were discouraged from reporting disease in compliance with the Communicable Disease Prevention Law.

In such circumstances, the Infectious Disease Control Law came into force as a means to emphasize the promotion of infectious disease prevention for society as a whole. As part of the efforts in such a shift, the new law underscored the importance of surveillance and provided the public and medical professionals with the information necessary to prevent infectious diseases based upon data from surveillance.

In the present study we describe the surveillance system and 
summarize the data reported before and after the overall reform of the surveillance system. An attempt was made to envisage the potential impact of the new surveillance system on disease reporting by comparing data under the new law with data under the old one.

No drastic changes, in other words, no break in continuity was observed in most of the diseases which listed in notifiable diseases in both periods, at least, in the diseases which were perceived as serious among the community in those days. Several reports have indicated that reporting completeness in the disease surveillance varied from $9 \%$ to $99 \%$ and was most strongly associated with the disease being reported. This may be related to the perceived seriousness of these diseases or to the greater financial and human resources devoted to treating and preventing them. ${ }^{6,7}$ Consistent with these observations, most of the notifiable diseases showed a similar incidence rate without any discontinuity of the data between the two periods.

Striking observations are found in the diseases which are perceived to be milder and non-life-threatening. Considerable gaps were noted for influenza, measles, pertussis and infectious diarrhea between the notifiable and sentinel reporting framework. As there were no major gaps throughout the overall observed period of the sentinel reporting framework, it is considered that data from sentinel reporting framework reflect the real situation more than those from the mandatory system. Although scarlet fever is not necessarily the same with group A streptococcal infection, it is curable by antibiotics and no longer life-threatening disease. Therefore, its surveillance is better on a sentinel system. Although no documented case definition was available under the old law, clinicians might have had a different perception of reporting criteria in these two systems.

One of the noteworthy features of the new surveillance system were disease categorization according to the disease impact and surveillance was correspondingly re-organized with the two different frameworks of mandatory and sentinel reporting. Through our current summary, the new surveillance system in combination with the mandatory system for severe diseases and sentinel system for milder diseases seems to be working better.

Although the characteristics of sentinels seem to have changed between the two periods, case reports per sentinels increased after the new law took effect. Exanthema subitum which is considered to be a standard disease to estimate the capture rate by existing sentinel clinics increased in the number of cases per sentinel. This might reflect the increased capture rate from re-designing the sentinel surveillance. But one must recall the increased general awareness of infectious diseases these days.

For most Japanese people, information about infectious diseases is not very familiar and sometimes difficult to understand. The index used in the sentinel system, the reported cases per sentinel, is not easy to understand. There have been several efforts to translate these data into more understandable expressions, and some of them are employed in the national system..$^{8-11}$

It is notable that the new law has clearly stated the importance of dissemination of information for determining the appropriate action to be taken. According to the new law, the national and prefectural/municipal infectious disease surveillance centers have been organized so that these institutions can play a central role in implementing surveillance and information dissemination. Many infectious disease surveillance centers including national one publish infectious disease reports weekly, monthly, and/or when necessary on the web and in document form. In addition to these regular reports, papers in the academic field serve to facilitate the effective feedback of information. ${ }^{12,13}$

The new law classifies target diseases by their health impact, and it appears to improve the overall surveillance performance as the purpose of surveillance becomes clearer, especially for disease perceived to be milder. But since a single surveillance system obviously can not satisfy all the needs for a wide range of infectious disease control activities, disease-specific analysis should be made for further evaluation of the surveillance system and tailoring more specific surveillance to specific objectives.

In conclusion, under the new law different surveillance schemes have been developed suitable to assess disease impact with documented reporting criteria along with the development of systematic information dissemination systems. But continuous efforts are warranted for evaluation and further improvement of the surveillance system and risk communication through ongoing research on data analysis and effective communication methods.

\section{REFERENCES}

1. Lederberg J, Shope RE, Oaks SC, eds. Emerging Infections: Microbial Threats to Health in the United States. National Academy Press, Washington, D.C., 1992.

2. Ministry of Health, Labour and Welfare of Japan. Chapter 6, Section 2. Measures against New Infectious Diseases, In the Annual Reports on Health and Welfare 1998-1999 Social Security and National Life. Available at http://www.mhlw.go.jp/ english/wp/wp-hw/vol1/p2c6s2.html. (accessed on 18 September 2007)

3. Infectious Disease Surveillance Center, National Institute of Infectious Diseases of Japan. Amendment of the Infectious Diseases Control Law, Japan. Byogen Biseibutsu Kenshutsu Joho Geppo 2004; 25: 1-3. (in Japanese)

4. Ministry of Health, Labour and Welfare of Japan. Case definitions for reporting in compliance with Infectious Disease Control Law. Available at http://www.mhlw.go.jp/bunya/ken kou/kekkaku-kansenshou11/01.html. (accessed on 18 September 2007) (In Japanese)

5. Hashimoto S, Murakami Y, Taniguchi K, Nagai M. Determination of the numbers of monitoring medical institutions necessary for estimating incidence rates in the surveillance of infectious diseases in Japan. Nippon Koshu Eisei Zasshi 1999; 46: 1068-77. (in Japanese)

6. Doyle TJ, Glynn MK, Groseclose SL. Completeness of notifiable infectious disease reporting in the United States: an 
analytical literature review. Am J Epidemiol 2002; 155: 86674.

7. Thacker SB, Choi K, Brachman PS. The surveillance of infectious diseases. JAMA 1983; 249: 1181-5.

8. Hashimoto S, Murakami Y, Taniguchi K, Nagai M. Detection of epidemics in their early stage through infectious disease surveillance. Int J Epidemiol 2000; 29: 905-10.

9. Hashimoto S, Murakami Y, Taniguchi K, Shindo N, Osaka $\mathrm{K}$, Fuchigami $\mathrm{H}$, et al. Annual incidence rate of infectious diseases estimated from sentinel surveillance data in Japan. J Epidemiol 2003; 13: 136-41.

10. Hashimoto S, Kawado M, Murakami Y, Tada Y, Shigematsu $\mathrm{M}$, Taniguchi $\mathrm{K}$, et al. Incidence of infectious diseases estimated by the surveillance data in 2002-2004 in Japan. Nippon Koshu Eisei Zasshi 2006; 53: 794-9. (in Japanese)
11. Murakami Y, Hashimoto S, Taniguchi K, Osaka K, Fuchigami H, Nagai M. Evaluation of a method for issuing warnings pre-epidemics and epidemics in Japan by infectious diseases surveillance. J Epidemiol 2004; 14: 33-40.

12. Murakami Y, Hashimoto S, Taniguchi K, Fuchigami H, Nagai M. Temporal and geographical variation in epidemics determined from the results of an infectious disease surveillance system in Japan, description of epidemic patterns by data-based criteria for epidemic periods. Nippon Koshu Eisei Zasshi 2000; 47: 925-35. (in Japanese)

13. Hashimoto S, Murakami Y, Taniguchi K, Osaka K, Shindo $\mathrm{N}$, Fuchigami H, et al. Observation of influenza epidemics by the alert system in the surveillance in 1999. Nippon Koshu Eisei Zasshi 2001; 48: 480-5. (in Japanese) 